\title{
Reviewing and interpreting bodies of evidence for preparing practice recommendations
}

Ian Askew

Population Council

Follow this and additional works at: https://knowledgecommons.popcouncil.org/departments_sbsr-rh

Part of the Quantitative, Qualitative, Comparative, and Historical Methodologies Commons How does access to this work benefit you? Let us know!

\section{Recommended Citation}

Askew, lan. 2013. "Reviewing and interpreting bodies of evidence for preparing practice recommendations," presentation at the HIP Technical Advisory Group Meeting, UNFPA, New York. 


\section{Reviewing and Interpreting \\ Bodies of Evidence for \\ Preparing Practice Recommendations}

Ian Askew

Co-Director, STEP UP Consortium

Population Council

Nairobi, Kenya

HIP Technical Advisory Group Meeting

$6-7^{\text {th }}$ June, 2013

UNFPA, New York 


\section{What types of evidence / knowledge to include in a review?}

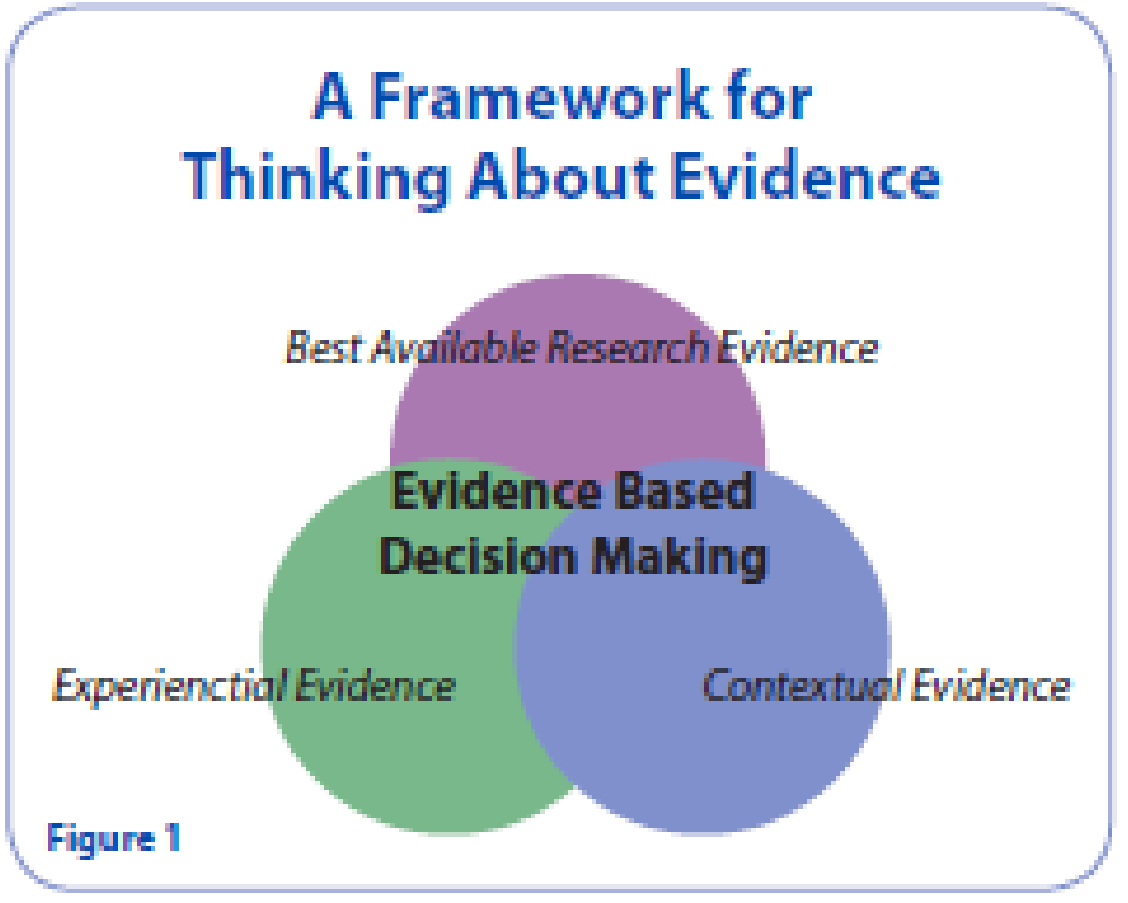

Puddy, R. W. \& Wilkins, N. (2011). Understanding Evidence Part 1: Best Available Research Evidence. A Guide to the Continuum of Evidence of Effectiveness. Atlanta: CDC

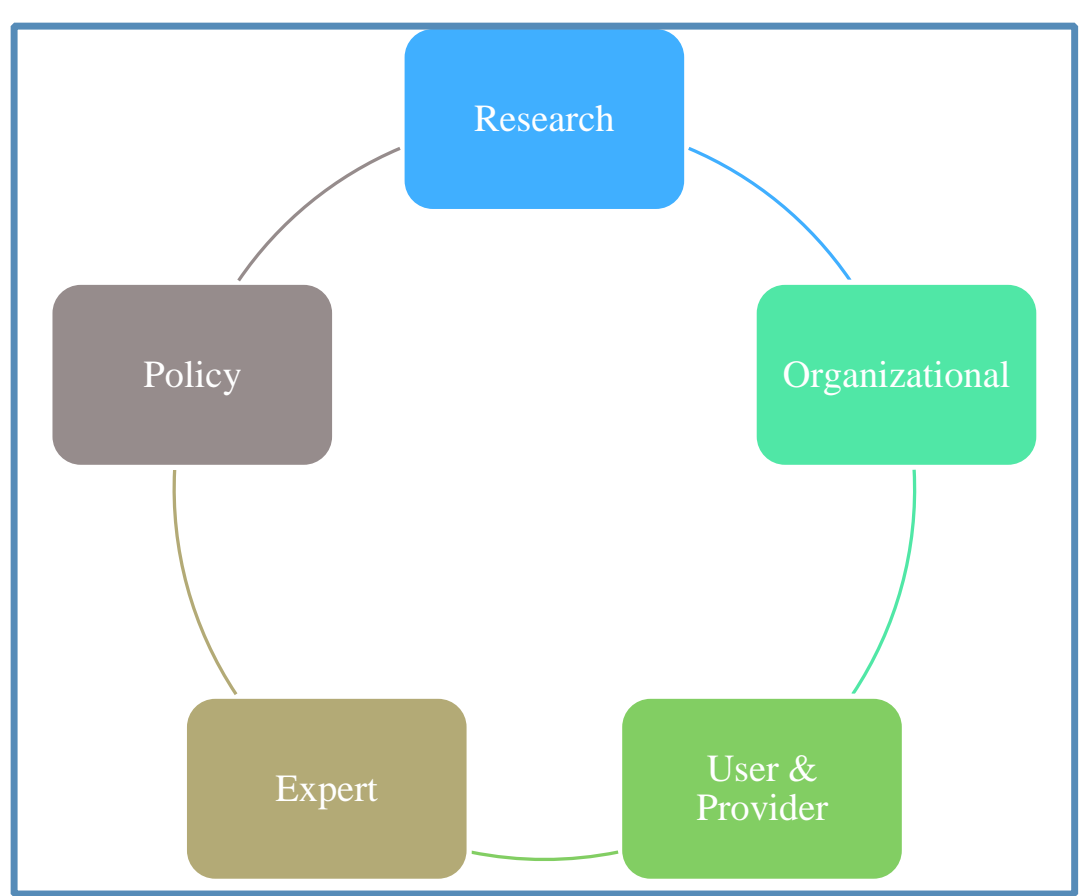

Rutter et al (2010). SCIE Systematic Research Reviews: Guidelines. London: Social Care Institute for Excellence
Consider also:

- "Practice enquiry"

- Economic consequences 


\section{Systematic Reviews}

"A systematic review is a scientific

investigation that focuses on a specific

question and uses explicit, pre-specified

scientific methods to identify, select, assess,

and summarize the findings of similar but

separate studies. It may include a quantitative

synthesis (meta-analysis), depending on the

available data"

(Institute of Medicine, US National Academy

of Sciences)

The key characteristics of a systematic review are:

- A clearly stated set of objectives with pre-defined eligibility criteria for including studies;

- An explicit, reproducible methodology;

- A systematic search that attempts to identify all studies that would meet the eligibility criteria;

- An assessment of the validity of the findings of the included studies;

- A systematic presentation, and synthesis, of the characteristics and findings of the included studies.

(Cochrane Review)

summary of the literature that uses as a explicit and systematic that uses identify, appraise and sumethods to literature according to predeterise the criteria. If this description (of the predeted methods) is not present, it is not po to make a thorough evaluation of thessible quality of the review" evaluation of the (UK National Institute for Health \& 


\section{Systematic review process}

-Step 1: Initiate the process:

-Step 2: Develop the review protocol:

-Step 3: Systematically locate, screen, and select the studies for review

-Step 4: Appraise the risk of bias in the individual studies and extract the data for analysis

-Step 5: Synthesize the findings and assess the overall quality of the body of evidence

-Step 6: Prepare a final report and have the report undergo peer review

Institute of Medicine 2011. Finding what works in health care: standards for systematic reviews, National Academy of Sciences 


\section{Criteria when assessing the overall strength of a body of evidence}

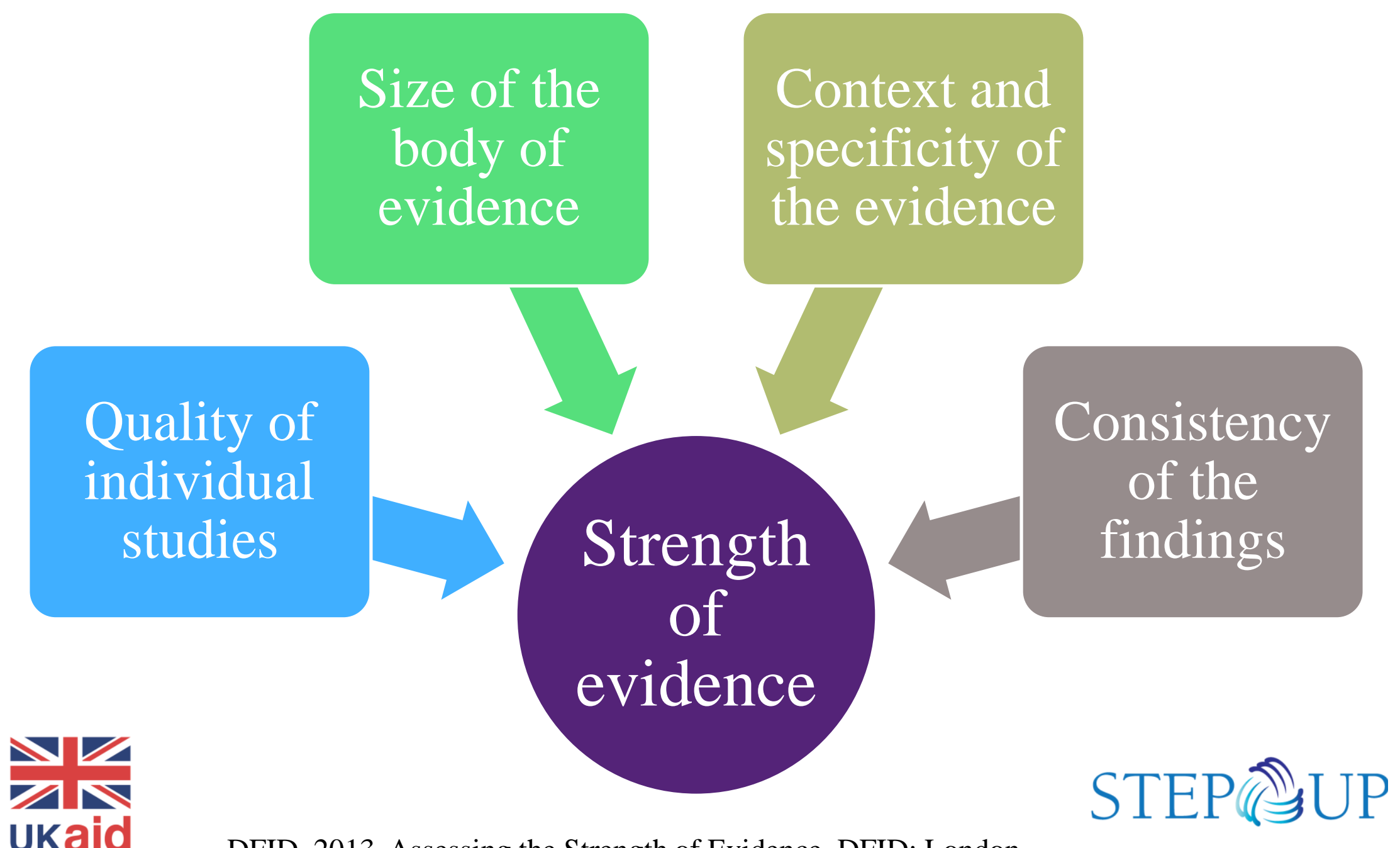

DFID. 2013. Assessing the Strength of Evidence, DFID: London 


\section{Categorizing the strength of evidence}

\section{Categories of Evidence}

\section{Combinations of Criteria}

High quality body of evidence, large in Very Strong size, consistent, and closely matched to the specific context of the intervention

High quality body of evidence, large or medium in size, generally consistent, and matched to the specific context of the Intervention

Moderate quality studies, medium size evidence body, generally consistent, which

Medium may or may not be relevant to the intervention. Limited number of high quality studies.

Moderate or low quality studies, small or

Limited medium size body, inconsistent, not matched to intervention

\section{Body of Evidence includes...}

Studies based on experimental designs (including impact evaluations), as well as systematic reviews and/or meta-analysis

Experimental or quasi-experimental designs, observational research designs that attempt counterfactual analysis, systematic reviews.

Multiple designs, but which have been assessed as being of moderate quality. The studies do not offer robust findings that can be derived and replicated across a range of contexts.

Varied designs and methodologies, which do not meet minimum standards. Includes causal inference from single case studies in limited contexts, and cross-sectional analysis without baseline data.

DFID. 2013. Assessing the Strength of Evidence, DFID: London 


\section{Using evidence to develop practice recommendations}

- GRADE emphasizes importance of separating quality of evidence from strength of recommendation

$>$ Strong recommendation for / against

$>$ Conditional recommendation

- Unanswered questions relating to effectiveness, safety, feasibility, acceptability: $\square$ "with rigorous research"

- Uncertainties about the intervention in certain conditions or contexts or populations: $\longrightarrow$ "with targeted M\&E" 


\section{Key issues}

$>$ Agreeing on types of knowledge to include and exclude

$>$ Agreeing on standards for a process of reviewing bodies of evidence

$>$ Hierarchy or matrix of evidence

$>$ Deriving strength of recommendation from quality of evidence

$>$ Terminology for describing evidence quality and strength of recommendation

$>$ Messaging, especially for "conditional" recommendations 

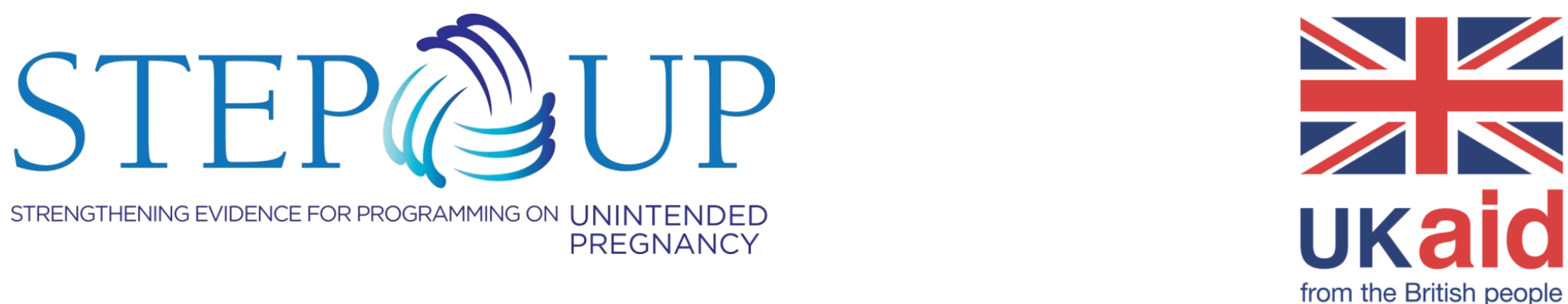

STRENGTHENING EVIDENCE FOR PROGRAMMING ON UNINTENDED

The STEP UP (Strengthening Evidence for Programming on Unintended Pregnancy) Research Programme Consortium is coordinated by the Population Council in partnership with the African Population and Health Research Center; icddr,b; the London School of Hygiene and Tropical Medicine; Marie Stopes International; and Partners in Population and Development. STEP UP is funded by UK aid from the UK Government.

\section{(2) Population Council}

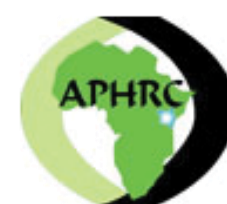
African Population and Health Research Center
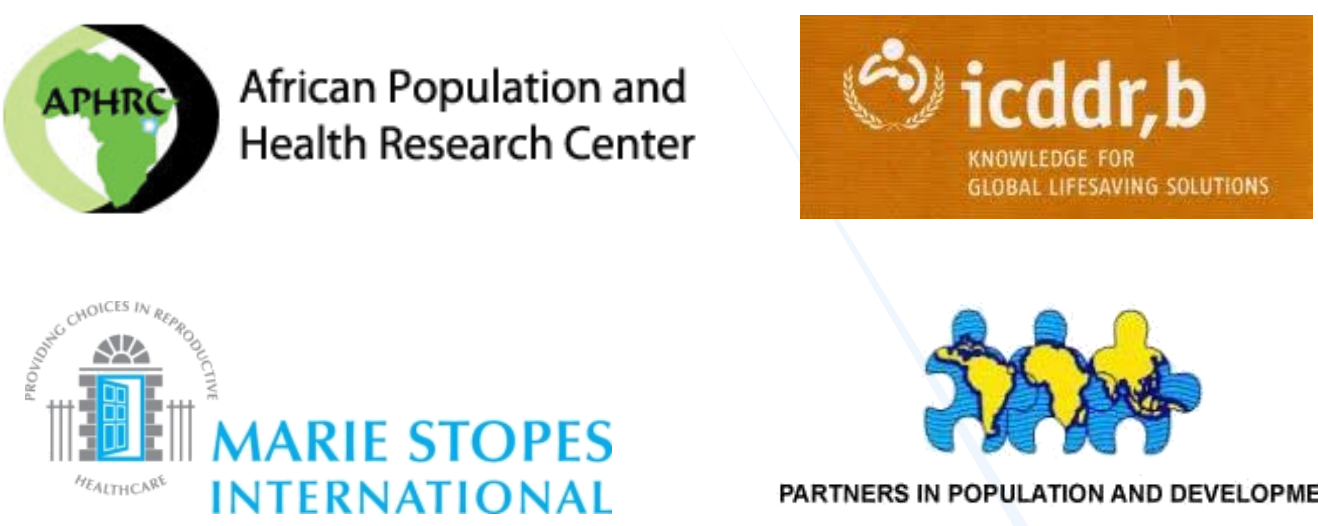

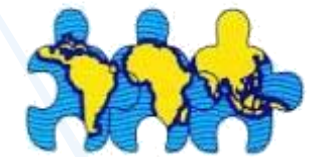

PARTNERS IN POPULATION AND DEVELOOPMENT
LONDON SCHOOL of HYGIENE MEDICINE

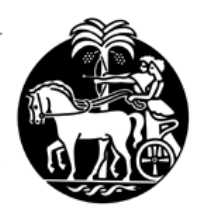

